\title{
Modified Ablation Index: A Novel Determinant of Successful First-Pass Left Atrial Posterior Wall Isolation
}

\author{
Satoshi Hayashida ${ }^{1}$, Koichi Nagashima ${ }^{1}$, Sayaka Kurokawa ${ }^{1}$, Masaru Arai ${ }^{1}$, Ryuta \\ Watanabe $^{1}$, Yuji Wakamatsu ${ }^{1}$, Naoto Otsuka ${ }^{1}$, Seina Yagyu ${ }^{1}$, Kazuki Iso ${ }^{2}$, and Yasuo \\ Okumura $^{1}$ \\ ${ }^{1}$ Nihon University School of Medicine \\ ${ }^{2}$ Kawaguchi Municipal Medical Center
}

August 11, 2020

\begin{abstract}
Introduction Although left atrial posterior wall isolation (LAPWI) in addition to pulmonary vein isolation is a well-accepted option for persistent atrial fibrillation (AF), complete isolation can be challenging. To evaluate performance of a modified ablation index (AI) (AI/bipolar voltage along the ablation line) for predicting durable LAPWI. Methods The study involved 55 consecutive patients, aged $65 \pm 11$ years, who underwent electroanatomic mapping-guided LAPWI for AF. Association between gaps (first-pass LAPWI failure and/or acute LAPW reconnections), voltage amplitude along the roof and floor lines, and thickness of the LAPW was investigated. Results Gaps occurred in 22 patients (40\%) and in 26 (8\%) of the 330 line segments assessed-11 in the center roof line segment, 6 in the center floor line segment, 4 in the right roof line segment, 4 in the right floor line segment, and 1 in the left floor line segment. Gaps were associated with relatively high bipolar voltage (3.38 \pm 1.83 vs. $1.70 \pm 1.12 \mathrm{mV}, \mathrm{P}<0.0001)$ and a thick $\mathrm{LA}$ wall $(2.52 \pm 1.15$ vs. $1.42 \pm 0.44 \mathrm{~mm}, \mathrm{P}<0.0001)$. A modified AI [?] $199 \mathrm{AU} / \mathrm{mV}$, bipolar voltage [?] $2.64 \mathrm{mV}$, wall thickness [?] $2.04 \mathrm{~mm}$, and roof ablation line [?] 43.4 mm well predicted gaps (AUCs: $0.783,0.787,0.858$, and 0.752, respectively). Conclusions High voltage zones, a thick LAPW, and a long roof ablation line appear to be determinants of gaps, and a modified AI [?] $199 \mathrm{AU} / \mathrm{mV}$ along the ablation lines appears to predict acute durable LAPWI.
\end{abstract}

\section{Modified Ablation Index: A Novel Determinant of Successful First-Pass Left Atrial Posterior Wall Isolation}

Satoshi Hayashida, $\mathrm{MD}^{1}$, Koichi Nagashima, MD, $\mathrm{PhD}^{1}$, Sayaka Kurokawa, MD, $\mathrm{PhD}^{1}$, Masaru Arai, MD, $\mathrm{PhD}^{1}$, Ryuta Watanabe, MD, $\mathrm{PhD}^{1}$, Yuji Wakamatsu, $\mathrm{MD}^{1}$, Naoto Otsuka, $\mathrm{MD}^{1}$, Seina Yagyu, $\mathrm{MD}^{1}$, Iso Kazuki, $\mathrm{MD}, \mathrm{PhD}^{2}$, Yasuo Okumura, $\mathrm{MD}, \mathrm{PhD}^{1}$

${ }^{1}$ Division of Cardiology, Department of Medicine, Nihon University School of Medicine, 30-1 Ohyaguchikamicho, Itabashi-ku, Tokyo 173-8610, Japan

${ }^{2}$ Division of Cardiology, Kawaguchi Municipal Medical Center, 180 Nishiaraijyuku, Kawaguchi-shi, Saitama 333-0833, Japan

Address for correspondence

Koichi Nagashima, MD, PhD; Division of Cardiology, Department of Medicine, Nihon University School of Medicine, 30-1 Ohyaguchi-kamicho, Itabashi-ku, Tokyo 173-8610, Japan

Tel: +81-3-3972-8111

Fax: +81-3-3972-1098 
E-mail: cocakochan@gmail.com

Total word count: 4373 words

Brief title: Modified AI as a Determinant of First-Pass LAPWI

The study was supported by departmental resources only, and the authors have no conflict of interest to declare.

\section{Abstract}

\section{Introduction}

Although left atrial posterior wall isolation (LAPWI) in addition to pulmonary vein isolation is a wellaccepted option for persistent atrial fibrillation ( $\mathrm{AF}$ ), complete isolation can be challenging.

To evaluate performance of a modified ablation index (AI) (AI/bipolar voltage along the ablation line) for predicting durable LAPWI.

\section{Methods}

The study involved 55 consecutive patients, aged $65 \pm 11$ years, who underwent electroanatomic mappingguided LAPWI for AF. Association between gaps (first-pass LAPWI failure and/or acute LAPW reconnections), voltage amplitude along the roof and floor lines, and thickness of the LAPW was investigated.

\section{Results}

Gaps occurred in 22 patients (40\%) and in $26(8 \%)$ of the 330 line segments assessed - 11 in the center roof line segment, 6 in the center floor line segment, 4 in the right roof line segment, 4 in the right floor line segment, and 1 in the left floor line segment. Gaps were associated with relatively high bipolar voltage (3.38 \pm 1.83 vs. $1.70 \pm 1.12 \mathrm{mV}, P<0.0001)$ and a thick LA wall $(2.52 \pm 1.15$ vs. $1.42 \pm 0.44 \mathrm{~mm}, P<0.0001)$. A modified AI [?] $199 \mathrm{AU} / \mathrm{mV}$, bipolar voltage [?] $2.64 \mathrm{mV}$, wall thickness [?] $2.04 \mathrm{~mm}$, and roof ablation line [?] $43.4 \mathrm{~mm}$ well predicted gaps (AUCs: $0.783,0.787,0.858$, and 0.752 , respectively).

\section{Conclusions}

High voltage zones, a thick LAPW, and a long roof ablation line appear to be determinants of gaps, and a modified AI [?] $199 \mathrm{AU} / \mathrm{mV}$ along the ablation lines appears to predict acute durable LAPWI.

Key Words: atrial fibrillation, left atrial posterior wall isolation, left atrial wall thickness, voltage map, ablation index

\section{Abbreviations}

AF, Atrial fibrillation; AI, Ablation index; ATP, Adenosine triphosphate; AUC, Areas under the curve; CF, Contact force; CT, Computed tomography; HVZs, High-voltage zones; LA, Left atrium; LAPWI, Left atrial posterior wall isolation; LIPV, Left inferior pulmonary vein; LSPV, Left superior pulmonary vein; PAF, Paroxysmal atrial fibrillation; PerAF, Persistent atrial fibrillation; PVI, Pulmonary vein isolation; RF, Radiofrequency; RIPV, Right inferior pulmonary vein; ROC, Receiver-operating characteristic; RSPV, Right superior pulmonary vein; 3D, three-dimensional.

\section{Introduction}

Left atrial (LA) posterior wall isolation (LAPWI) added to pulmonary vein isolation (PVI) can improve ablation outcomes for persistent atrial fibrillation (PerAF) by modifying ganglionated plexi, debulking LA tissue including the substrate and rotors, and reinforcing the results of PVI. ${ }^{1}$ Sometimes LAPWI is challenging, however, requiring numerous high-power radiofrequency $(\mathrm{RF})$ energy applications. Residual posterior wall conduction can exist after first-pass LAPWI and may be the result of failure to create transmural lesions or of gaps in the LA roof or floor line, explaining, at least in part, the questionable efficacy of this strategy. ${ }^{2-4}$ Ablation index (AI)-guided high-power short-duration ablation in which the catheter-tip contact force (CF), 
output power, and duration of RF application are monitored is a well-developed strategy for the creation of durable lesions and has substantially improved ablation outcomes in patients with $\mathrm{AF}^{5-7}$ Therefore, in clinical practice, first-pass AI-guided LAPWI can be expected.

We have reported high-voltage zones (HVZs) within the PV-encircling lines to be a major determinant of acute PV reconnections after PVI; the HVZs might reflect thickened wall or non-diseased cardiomyocytes, which can impede the creation of transmural lesions.${ }^{8,9}$ Based on these findings, we proposed a novel index, the "modified AI", calculated as AI/bipolar voltage at the target ablation site, and showed this index to be useful in predicting acute durable PVI. ${ }^{8}$ We have since hypothesized that HVZs and a thickened LA wall are obstacles to complete LAPWI, and, in this study, we evaluated association between gap locations after first-pass LAPWI and local preprocedural bipolar voltage and computed tomography (CT)-based measured LA wall thickness under the ablation line, and we determined the modified AI predictive of acute durable LAPWI.

\section{Methods}

\subsection{Study patients}

Included in our study were 55 patients with $\mathrm{AF}$ (38 men and 17 women who ranged in age from 35 to 85 years). Forty-seven of the patients had PerAF, and 8 had paroxysmal AF (PAF). All had undergone LAPWI after PVI at either Nihon University Itabashi Hospital or Kawaguchi Municipal Medical Center between August 2019 and June 2020. For 12 of the 55 patients (4 with PerAF and 8 with PAF), LAPWI had been performed due to AF recurrence after PVI. For the purpose of the study, we divided the patients into 2 groups: those for whom first-pass LAPWI was successful without acute LAPW reconnection $(\mathrm{n}=33$, Successful first-pass group) and those for whom first-pass LAPWI was not successful and/or acute LAPW reconnections were seen $(\mathrm{n}=22$, Gap group). The study was approved by Nihon University Itabashi Hospital Clinical Research Judging Committee and Kawaguchi Municipal Medical Center Ethics Committee.

\subsection{CT-based measurement of LAPW thickness}

Multidetector helical CT had been performed with a 320-row detector, dynamic volume CT scanner (Aquilion ONE; Toshiba Medical Systems, Tokyo, Japan) before the ablation procedure. Scanning was performed at a slice thickness of $0.5 \mathrm{~mm}$, gantry rotation time of $350 \mathrm{~ms}$, tube voltage of $120 \mathrm{kV}$, and tube current of 300-580 $\mathrm{mA}$ for optimum detection of fine structures (resolution of approximately $0.3 \mathrm{~mm}$ ). Patients' heart rate $<65$ bpm was maintained by Landiolol administration, and nonionic iodinated contrast medium (Iomeron, Eisai Co, Tokyo, Japan) was injected at $0.07 \mathrm{~mL} / \mathrm{kg} / \mathrm{second}$ for 9 seconds. Timing of the image acquisition was determined by means of bolus tracking software; imaging was initiated when contrast reached the LA. During the end-expiratory phase, the volume acquisition was gated to $65 \%-75 \%$ of the R-R interval on the lead II electrocardiogram during sinus rhythm or AF rhythm. Thickness of the LAPW was measured as previously described..$^{9-11}$ The acquired CT images were transferred to a workstation (ZIO M900 3.0; QUADRA: Amin Co., Ltd., Tokyo, Japan). Hounsfield units (HUs) of -50 to -200 were assigned to detect epicardial fat and exclude it from the LAPW. ${ }^{9-13}$ Thickness of the LAPW was measured at the roof and floor lines, with each line was divided into 3 segments: right, center, and left segments. Coronal plane images were used to measure wall thickness at the roof line and horizontal plane images to measure wall thickness at the floor line (Figure 1). Wall thickness was measured at 3 sites per segment, the average of the 3 values was calculated for each segment, and these average values were used for analysis. ${ }^{9-11}$

\subsection{Electrophysiologic study and voltage mapping}

All antiarrhythmic drugs were discontinued for at least 5 half-lives before the ablation procedure. Electrophysiologic study was undertaken with patients under conscious sedation achieved with dexmedetomidine, propofol, and fentanyl.,9 Two long sheaths (Agilis steerable sheath and SL0 sheath, St. Jude Medical Inc., St. Paul, MN) were positioned in the LA. Heparin was used to maintain an activated clotting time $>300$ seconds throughout the procedure.

For each patient, a high-density LA voltage map was created by means of a 3-dimensional mapping system 
(CARTO3, Biosense Webster, Diamond Bar, CA) and a multispline catheter with 2-mm interelectrode spacing (PentaRay NAV; Biosense Webster). The map was created during sinus rhythm, and if before ablation the patient was in AF rhythm, the map was created after sinus rhythm was restored by means of low-energy (10-20 joules) internal cardioversion performed with a catheter (BeeAT; Japan Lifeline, Tokyo, Japan) placed in the coronary sinus. Bipolar signals were acquired with a high-pass filter at $30 \mathrm{~Hz}$ and a low-pass filter at $500 \mathrm{~Hz}$. For each of the 3 segments on the roof line and each of the 3 segments on the floor line where the CT-based measurements of LAPW thickness were obtained, voltage was measured at 3 sites closest to the ablation line, and the 3 measured voltages were averaged for the analysis (Figure 2). Obviously dull potentials, suggesting the electrograms were recorded by the catheter not in contact with the LA, were excluded. In addition, the modified AI was calculated for each of the 3 segments on both ablation lines.

\subsection{Ablation procedure and confirmation of LAPWI}

LAPWI was performed after confirmation of bilateral PVI. RF energy was applied for 15 seconds with a 3.5mm open-irrigated-tip catheter (Navistar ThermoCoolSmartTouch SF; Biosense Webster) set at an upper temperature limit of $43 \mathrm{degC}$, a saline irrigation flow rate of $17-30 \mathrm{~mL} / \mathrm{min}$ (CoolFlow Pump; Biosense Webster), and output power of $45 \mathrm{~W}$. In all cases, automated ablation lesion tagging based on catheter stability information (VisiTag Module, CARTO3) was used, but the RF delivery time was not guided by AI. The RF power output was reduced to $25-35 \mathrm{~W}$ when the esophageal temperature rose, and RF energy delivery was terminated if the probe-monitored esophageal temperature reached 40degC. Complete LAPWI was confirmed by bidirectional block. At least 30 minutes after the procedure, $30 \mathrm{mg}$ of adenosine triphosphate (ATP) was injected to confirm persistent roof and floor line block. ${ }^{14}$

If the first-pass LAPWI failure or if acute LAPW reconnection, defined as spontaneous reconnection or dormant conduction provoked by ATP, was seen after LAPWI was attempted, the segment with a gap in the block line was identified by the PentaRay catheter; noted as a right, center, or left segment; and subjected to additional RF energy application. Successful first-pass LAPWI was defined as complete LAPWI (absence of acute LAPW reconnections) achieved during the initial RF applications.

\subsection{Variables analyzed}

The following information was obtained from patients' records and compared between the 2 study groups: patients' clinical characteristics at the time of the procedure, e.g., age, sex, presence of diabetes mellitus, $\mathrm{CHADS}_{2}$ and $\mathrm{CHA}_{2} \mathrm{DS}_{2}$-VASc scores, creatinine and NT-proBNP concentrations, and echocardiographic variables plus patients' CT-based measured wall thicknesses and variables related to the ablation procedure. Also compared was the modified AI for segments with a gap in the ablation line vs. that for segments without a gap in the ablation line.

\subsection{Statistical analysis}

Continuous variables are expressed as mean +- SD values or median, and categorical variables are expressed as the number and percentage of patients. Student'st -test or Mann-Whitney $U$ test was used, as appropriate, to analyze between-group differences in continuous variables, and the chi-square test was used to analyze between-group differences in dichotomous variables unless the expected counts were $<5$, in which case Fisher's exact test was used. Receiver operating characteristic (ROC) curves were plotted to determine the cutoff values of the ablation-related variables for prediction of gaps. The areas under the curve (AUC) were compared by DeLong test. The ROC curves and AUCs were analyzed with MedCalc ver. 16.4.1 (MedCalc Software, Mariakerke, Belgium). All other statistical analyses were performed with JMP 11 software (SAS Institute, Cary, NC). $P<0.05$ was considered statistically significant.

\section{Results}

\subsection{Characteristics of patients, per study group}

Characteristics of patients are shown per study group in Table 1. Overall, LAPWI was achieved in 54 of 
the 55 patients (98\%). LAPWI was not achieved in 1 patient (2\%) despite numerous high-power RF energy applications. For 50 patients in whom LAPWI was achieved, RF energy was applied only to the LA roof and floor, but 5 patients required additional RF application at the center of the LAPW or interior surface of the LA. With the exception of body weight, patient characteristics, echocardiographic variables, and serologic test results did not differ significantly between the Successful first-pass group and the Gap group. Body weight was greater in the Gap group.

\subsection{Electrophysiologic and CT-based findings, per study group}

On voltage mapping, $1759+-767$ points were acquired per patient. LAPWI required $34+-12$ ablation points: $16+-7$ for the roof line and $17+-5$ for the floor line. Gaps were detected in 22 patients (40\%), with 22 gaps due to first-pass LAPWI failure and 4 due to dormant conduction (Figure 3). A representative case of a gap in the center of the roof line lesion is shown in the Figures 1 and 2. The electrophysiologic features and LAPW thicknesses are shown per group in Table 2. Overall, mean bipolar voltage along the ablation line and mean LAPW thickness were greater in the Gap group than in the Successful first-pass group. The roof line was longer, and mean bipolar voltage and thickness of the LA wall at the roof line were greater in patients with a gap in the roof line than in other patients. However, no differences were seen in the floor line. Significant correlation was found between LAPW thickness and bipolar voltage $(\mathrm{r}=0.25, P<0.0001)$ (Figure 4).

\subsection{Gap characteristics per segment}

Distribution of the gaps and features of the segments with gaps are shown in Figure 3 and Table 3, respectively. Gaps were observed in 26 (8\%) of the 330 line segments assessed; 11 in the center roof line segment, 6 in the center floor line segment, 4 in the right roof line segment, 4 in the right floor line segment, and 1 in the left floor segment. The gaps were observed in segments of higher voltage $(3.38+-1.83$ vs. $1.70+-1.12 \mathrm{mV}$, $P<0.0001)$, with a thicker LA wall $(2.52+-1.15$ vs. $1.42+-0.44 \mathrm{~mm}, P<0.0001)$. However, no differences were found in the number of RF points, presence of inter-lesion distance [?] $6 \mathrm{~mm}$, impedance drop during RF application, or AI. The modified AI was lower in the segments with a gap than in the isolated segments (124 [94-198] vs. 288 [182-463] AU/mV, $P<0.0001)$.

A 3DCT image and LA bipolar voltage map obtained in a case in which LAPWI was not achieved after numerous high-power RF energy applications are shown in Figure 5. The roof line was found to be incomplete, the wall at the center of the roof line (panel A) was very thick, and the voltage (panel B) was extremely high.

\subsection{Performance of the bipolar voltage amplitudes at the roof and floor lines, LAPW thickness,} and modified AI for prediction of acute LAPW reconnection

The ROC curve drawn for LA roof line length showed the best cutoff value (AUC: 0.752 ) for predicting presence of a gap in the roof line to be [?] $43.4 \mathrm{~mm}$, with a sensitivity of $50 \%$ and specificity of $95 \%$. The ROC curve drawn for bipolar voltage showed the best cutoff value (AUC: 0.787 ) for predicting a gap resulting from the initial attempt at the LAPWI to be [?] $2.64 \mathrm{mV}$ (sensitivity: $69 \%$; specificity: $83 \%$ ), and the curve drawn for LAPW thickness (AUC: 0.858) showed the best cutoff value to be [?] $2.04 \mathrm{~mm}$ (sensitivity: $65 \%$; specificity: 93\%) (Figure 6). For the modified AI, the best cutoff value (AUC: 0.783 ) for predicting presence of a gap was [?] $199 \mathrm{AU} / \mathrm{mV}$ (sensitivity: $81 \%$; specificity: $70 \%$ ).

\section{Discussion}

\subsection{Main findings}

The main findings of our study were as follows: (1) A gap following attempted LAPWI was often located either at the center or right aspect of the line; (2) HVZs [?] $2.64 \mathrm{mV}$ and an LA wall [?] $2.04 \mathrm{~mm}$ in thickness at the ablation line, and a long roof ablation line [?] $43.4 \mathrm{~mm}$ were predictive of a gap in the ablation line; and (3) the best cutoff modified AI value for predicting acute durable LAPWI was [?] $199 \mathrm{AU} / \mathrm{mV}$. 
Whether LAPWI provides additional benefits over PVI alone is controversial. ${ }^{2-4,15}$ Achievement of complete LAPWI without additional ablation within the lines is only modest $(45 \%-72 \%),{ }^{15}$ which might hinder the efficacy of the procedure. Incomplete LAPWI may result from failure to create transmural lesions in the roof line or floor line. To overcome this problem, several new technologies such as AI and high-power short duration ablation have been developed. These modalities are aimed at improving the "operator factors" by generalizing and standardizing the physicians' skills and experience as applied to the ablation procedure. However, ablation lesions can also be influenced by "patient factors," such as the wall thickness and tissue characteristics, e.g., diseased vs. healthy tissue. Results of our previous studies have shown that a high bipolar voltage area [?] $2.69 \mathrm{mV}$ and a thick LA wall were obstacles to achievement of complete PVI, and the modified AI, which takes into account both operator and patient factors, of [?] $189 \mathrm{AU} / \mathrm{mV}$ was found to predict acute durable PVI. ${ }^{8}$ In the study reported herein, we obtained similar results, with our data indicating that the modified AI can also be applied to LAPWI.

Among our study patients, average LAPW thickness varied, ranging from $0.6 \mathrm{~mm}$ to $5.5 \mathrm{~mm}$. The center of the roof line lies above the thickest part, measuring up to $6.5 \mathrm{~mm}$. This is because the septopulmonary bundle runs behind Bachmann's bundle and passes over the roof. ${ }^{16,17}$ Other investigators have reported that gaps were frequently located at the center and right of the roof line because of lower stability and thicker tissue with interatrial muscular bridges. ${ }^{16,18}$ Results of our study are consistent with these reported findings. Furthermore, bipolar voltage is reported to be a surrogate marker of LA wall thickness. ${ }^{9}$ Although wall thickness is also a good predictor of acute PV reconnections, ${ }^{11}$ it is not informative with respect to tissue characteristics. Real-time assessment and accurate measurement of the thickness of the wall just underneath the ablation tags are also limited due to the technical difficulties of incorporating such information into a 3D mapping system. Bipolar voltage is a simple real-time variable obtained at sites accurately mapped in 3D.

In contrast, another major obstacle to creating a continuous floor line lesion would be its proximity to the esophagus, frequently forcing a limit on the ablation power or duration to avoid esophageal temperature rises. ${ }^{15}$ However, we found no association between gaps and sites where the esophageal temperature rose. A gap in the floor line might be due simply to catheter instability.

One major concern is that patient factors cannot be adjusted by the operators. Therefore, an AI that accounts for both the operator and patient factors is needed for predicting durable LAPWI. With the goal of producing durable but non-excessive ablation, we developed a modified AI, which accounts for both operator and patient factors. We intended to deliver the RF energy with same output, duration, and contact force resulting in the same AI to all the lesion for the calculation of the modified AI. We found the modified AI to be of good prognostic performance for predicting gaps in the ablation lines when attempting LAPWI, and we found the best cutoff value to be [?] $199 \mathrm{AU} / \mathrm{mV}$. For example, this cutoff value indicated that an AI of [?] $398 \mathrm{AU}$ will be needed if bipolar voltage at the target ablation site is $2 \mathrm{mV}$ and an AI of [?] $597 \mathrm{AU}$ for $3 \mathrm{mV}$. The modified AI-guided ablation strategy considering bipolar voltage might be a potential ablation method to increase the acute lesion durability and reduce the procedure time and complications. Repeated ablation sessions for acute PV reconnections are time-consuming and may produce tissue edema that can cause failure of transmural lesions. ${ }^{19}$ Further prospective studies are needed to clarify the effectiveness of modified AI-guided LAPWI.

\subsection{Study limitations}

Our study findings should be interpreted in light of our study limitations, the first of which is its execution as a study involving a fairly small number of patients. Second, the recorded bipolar voltages might have been affected by various factors, such as catheter orientation, contact force, electrode area, and interelectrode spacing. We note, however, that the best performing currently available mapping catheter was used. Third, gaps might have been caused by various other factors, such as the catheter contact angle, circulating blood flow, and trabecula in the LA. All of these factors need to be considered to ensure lesion durability.

\section{Conclusion}

Areas of high voltage (i.e., [?] $2.64 \mathrm{mV}$ ) and thick LA walls (i.e., [?] $2.04 \mathrm{~mm}$ ) under the ablation line, and 
a long roof line [?] $43.4 \mathrm{~mm}$ were shown to be determinants of conduction through gaps in the ablation line after first-pass LAPWI. A modified AI [?] 199 AU/mV may predict durable LAPWI.

References

1. Calkins H, Kuck KH, Cappato R, et al. 2012 HRS/EHRA/ECAS Expert Consensus Statement on Catheter and Surgical Ablation of Atrial Fibrillation: recommendations for patient selection, procedural techniques, patient management and follow-up, definitions, endpoints, and research trial design. Europace . 2012; 14 (4): 528- 606.

2. Bai R, Di Biase L, Mohanty P, et al. Proven isolation of the pulmonary vein antrum with or without left atrial posterior wall isolation in patients with persistent atrial fibrillation. Heart Rhythm . 2016; 13 (1): $132-140$.

3. Lee JM, Shim J, Park J, et al. The electrical isolation of the left atrial posterior wall in catheter ablation of persistent atrial fibrillation. JACC Clin Electrophysiol. 2019; 5 (11): 1253- 1261.

4. Sutter JS, Lokhnygina Y, Daubert JP, et al. Safety and efficacy outcomes of left atrial posterior wall isolation compared to pulmonary vein isolation and pulmonary vein isolation with linear ablation for the treatment of persistent atrial fibrillation. Am Heart J . 2020;220 : 89- 96.

5. Winkle RA, Mohanty S, Patrawala RA, et al. Low complication rates using high power (45-50 W) for short duration for atrial fibrillation ablations. Heart Rhythm. 2019; 16 (2): 165- 169.

6. Okamatsu H, Koyama J, Sakai Y, et al. High-power application is associated with shorter procedure time and higher rate of first-pass pulmonary vein isolation in ablation index-guided atrial fibrillation ablation. $J$ Cardiovasc Electrophysiol. 2019; 30 (12): 2751- 2758.

7. Phlips T, Taghji P, El Haddad M, et al. Improving procedural and one-year outcome after contact forceguided pulmonary vein isolation: the role of interlesion distance, ablation index, and contact force variability in the 'CLOSE'-protocol. Europace. 2018;20 (FI_3): f419- f427.

8. Wakamatsu Y, Nagashima K, Watanabe I, et al. The modified ablation index: a novel determinant of acute pulmonary vein reconnections after pulmonary vein isolation. J Interv Card Electrophysiol . 2019;55 (3): $277-285$.

9. Nagashima K, Watanabe I, Okumura Y, et al. High-voltage zones within the pulmonary vein antra: major determinants of acute pulmonary vein reconnections after atrial fibrillation ablation. J Interv Card Electrophysiol . 2017; 49 (2): 137- 145.

10. Takahashi K, Okumura Y, Watanabe I, et al. Relation between left atrial wall thickness in patients with atrial fibrillation and intracardiac electrogram characteristics and ATP-provoked dormant pulmonary vein conduction. J Cardiovasc Electrophysiol . 2015;26 (6): 597- 605.

11. Iso K, Okumura Y, Watanabe I, et al. Wall thickness of the pulmonary vein-left atrial junction rather than electrical information as the major determinant of dormant conduction after contact force-guided pulmonary vein isolation. J Interv Card Electrophysiol . 2016;46 (3): 325- 333.

12. Nagashima K, Okumura Y, Watanabe I, et al. Association between epicardial adipose tissue volumes on 3-dimensional reconstructed CT images and recurrence of atrial fibrillation after catheter ablation. Circ $J$ . 2011; 75 (11): 2559- 2565.

13. Nagashima K, Okumura Y, Watanabe I, et al. Does location of epicardial adipose tissue correspond to endocardial high dominant frequency or complex fractionated atrial electrogram sites during atrial fibrillation? Circ Arrhythm Electrophysiol. 2012; 5 (4): 676- 683.

14. McLellan AJA, Prabhu S, Voskoboinik A, et al. Isolation of the posterior left atrium for patients with persistent atrial fibrillation: routine adenosine challenge for dormant posterior left atrial conduction improves long-term outcome. Europace. 2017; 19 (12): 1958- 1966. 
15. Thiyagarajah A, Kadhim K, Lau DH, et al. feasibility, safety, and efficacy of posterior wall isolation during atrial fibrillation ablation. Circ Arrhythm Electrophysiol. 2019; 12 (8): e007005.

16. Ho SY, Sanchez-Quintana D, Cabrera JA, Anderson RH. Anatomy of the left atrium: implications for radiofrequency ablation of atrial fibrillation. Journal of cardiovascular electrophysiology . 1999;10 (11): 1525- 1533.

17. Markides V, Schilling RJ, Ho SY, Chow AW, Davies DW, Peters NS. Characterization of left atrial activation in the intact human heart.Circulation. 2003; 107 (5): 733- 739.

18. Wolf M, El Haddad M, Fedida J, et al. Evaluation of left atrial linear ablation using contiguous and optimized radiofrequency lesions: the ALINE study. Europace. 2018; 20 (FI_3): f401- f409.

19. Arujuna A, Karim R, Caulfield D, et al. Acute pulmonary vein isolation is achieved by a combination of reversible and irreversible atrial injury after catheter ablation: evidence from magnetic resonance imaging. Circ Arrhythm Electrophysiol . 2012; 5 (4): 691- 700.

\section{Figure legends}

Figure 1 Representative measurements of the roof (left panel) and floor line (right panel) on 3-dimentional computed tomography (3DCT) images in a case in which a gap was detected at the center of the roof line. Coronal plane images are used for measurement of the roof line, and horizonal plane images for measurement of the floor line. Each line is divided into 3 segments; right, center, and left segments (red lines). Ao = aorta; LA = left atrium; LIPV = left inferior pulmonary vein; LSPV = left superior pulmonary vein; $\mathrm{LV}=$ left ventricle; RIPV = right inferior pulmonary vein; RSPV = right superior pulmonary vein.

Figure 2 Representative left atrial bipolar voltage map from a case in which a gap was detected at the center of the roof line. Purple areas on the map are high voltage zones ([?] $2.64 \mathrm{mV}$ ), and blue, green, yellow, orange, and red areas are those of lower voltage $(<2.64 \mathrm{mV})$. LIPV = left inferior pulmonary vein; LSPV $=$ left superior pulmonary vein; RIPV $=$ right inferior pulmonary vein; RSPV $=$ right superior pulmonary vein.

Figure 3 Histograms showing average bipolar voltage (upper panel) at each of the 6 segments in which voltage was measured and left atrial posterior wall (LAPW) thickness (lower panel) at each of the same 6 segments. Open circles below the upper histogram indicate failed first-pass LAPW isolation (LAPWI), solid circles indicate acute LAPW reconnections.

Figure 4 Scatter plot and regression line showing the relation between left atrial bipolar voltage and left atrial posterior wall (LAPW) thickness.

Figure 5 Three-dimensional computed tomography image (3DCT) and LA bipolar voltage map obtained in a case in which first-pass left atrial posterior wall isolation (LAPWI) was unsuccessful due to a gap in the center of the roof line. As seen, the LA wall (A) is $5.41 \mathrm{~mm}$ thick, and the LA bipolar voltage (B) is $3.63 \mathrm{mV} . \mathrm{LA}=$ left atrium; LIPV = left inferior pulmonary vein; LSPV = left superior pulmonary vein; $\mathrm{LV}=$ left ventricle; $\mathrm{PA}=$ pulmonary artery; $\mathrm{RIPV}=$ right inferior pulmonary vein; $\mathrm{RSPV}=$ right superior pulmonary vein.

Figure 6 Receiver-operating characteristic curves for prediction of a gap after an initial attempt at left atrial posterior wall isolation (LAPWI). The modified AI, the bipolar voltage and wall thickness on the LAPWI line provide significantly better predictive value than the AI $(\mathrm{P}<0.01$, respectively $)$. AI $=$ ablation index.

\section{Hosted file}

table new.docx available at https://authorea.com/users/349770/articles/474742-modifiedablation-index-a-novel-determinant-of-successful-first-pass-left-atrial-posterior-wallisolation 


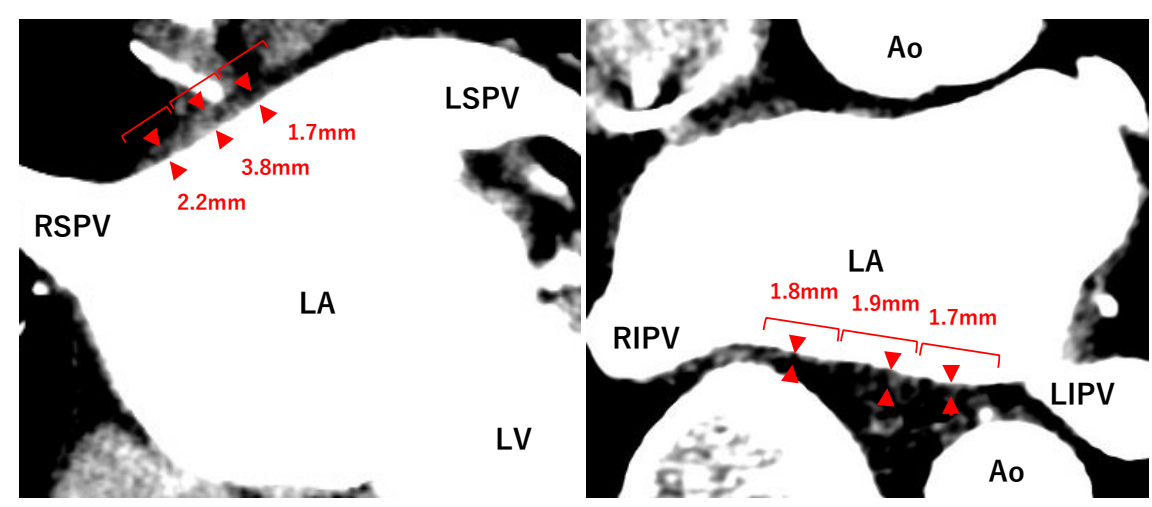

\section{Hosted file}

figure new.pdf available at https://authorea.com/users/349770/articles/474742-modifiedablation-index-a-novel-determinant-of-successful-first-pass-left-atrial-posterior-wallisolation 